\title{
SN38 increases IL-8 expression through the MAPK pathways in HCT8 cells
}

\author{
LEI ZHANG, WEN-HUI LOU, XUE-FENG XU, WENCHUAN WU, YE-FEI RONG and DA-YONG JIN \\ Pancreatic Cancer Group, General Surgery Department, Zhongshan Hospital, Fudan Universitiy, Shanghai 200232, P.R. China
}

Received February 9, 2016; Accepted November 9, 2016

DOI: $10.3892 /$ ijmm.2016.2810

\begin{abstract}
The overexpression of interleukin-8 (IL-8) is closely associated with poor tumor differentiation, metastasis and tumor progression. This study aimed to examine the effects and mechanisms of action of SN38 (a metabolite of the camptothecin derivative, CPT-11) on IL-8 expression in HCT8 cells, using ELISA, CCK-8 and western blot analysis. Among jatrorrhizine, evodiamine, 5-fluorouracil and SN38, SN38 was found to inhibit the proliferation of HCT8 cells in a dose-dependent manner, but to increase IL-8 secretion from HCT8 cells. Of the other agents, evodiamine was found to inhibit both IL-8 secretion and cell proliferation, and jatrorrhizine was found to increase IL-8 secretion without any obvious inhibitory effect on cell proliferation. Further experiments revealed that the increased activation of p38 mitogen-activated protein kinase (MAPK), extracellular signal-regulated kinase (ERK)1/2 and c-Jun N-terminal kinase (JNK) by SN38 contributed to the decreased cell proliferation and to the overexpression of IL-8 induced by SN38. Our results suggested that the MAPK pathways are activated by SN38, resulting in the upregulation of IL- 8 expression and in the inhibition of cell proliferation in an IL-8-independent manner. Thus, the potential benefit of the use of a combination of camptothecin-11 with other chemical drugs with inhibitory effects on IL-8 expression, should be paid more attention in treating colon cancer.
\end{abstract}

\section{Introduction}

Interleukin-8 (IL-8), a cytokine of the CXC chemokine family (1), is highly expressed in many tumor tissues, but is expressed at low levels in normal tissues (2) and promotes tumor progression, tissue invasion, metastasis and angiogenesis (3-10). In colon cancer, IL-8 has found to be markedly overexpressed in the majority of tumor tissues, and to be closely associated with poor tumor differentiation, metastasis and tumor progression (11). The neutralization of IL- 8 may suppress the invasive potential

Correspondence to: Dr Da-Yong Jin, Pancreatic Cancer Group, General Surgery Department, Zhongshan Hospital, Fudan University, 180 Fenlin Road, Shanghai 200232, P.R. China

E-mail: jin.dayong@zs-hospital.sh.cn

Key words: interleukin-8, camptothecin-11, colon cancer, evodiamine, mitogen-activated protein kinase, SN38 of colonic tumors in vivo (12). Moreover, the elevated expression of IL-8 is closely associated with the increased adhesion, migration and invasion of human gastric cancer cells $(1,3,7)$. The stable overexpression of IL-8 increases cell adhesion, migration and invasion, whereas the knockdown of IL-8 induces opposite effects (1). Mice expressing human IL-8 exhibit enhanced inflammation and accelerated colon carcinogenesis (8).

The overexpression of IL- 8 in tumor cells is often induced in response to chemotherapeutic agents (e.g., 5-fluorouracil, adriamycin, dacarbazine and paclitaxel) (13-17). Camptothecin-11 (CPT-11), a water-soluble semi-synthetic derivative of CPT with less toxic effects, is often used in the treatment of many types of solid tumors, including those of the lung, colon/rectum, ovaries and gastric system in clinic practice $(18,19)$. SN38, the active metabolite of CPT-11, has been shown to induce the upregulation of IL- 8 in the gastric cancer cell line, AGS (16). However, the effects of SN38 on IL-8 expression in colon cancer cells have not been elucidated to date, at least to the best of our knowledge. In addition, the molecular mechanisms responsible for the increase in IL-8 expresssion induced by SN38 have not yet been determined.

Mitogen-activated protein kinases (MAPKs) are known to play a role in cell proliferation and IL-8 secretion $(1,9,10)$. Berberine hydrochloride has been shown to inhibit cancer cell proliferation and IL- 8 secretion by inhibiting the activation of the MAPK pathway $(9,10)$. However, evodiamine has been shown to inhibit cancer cell proliferation but enhance IL-8 expression by promoting MAPK activation (10). However, whether other chemotherapeutic drugs can also enhance IL-8 secretion in colon cancer cells has not yet been clarified.

Therefore, in present study, the effects of SN38 on colon cancer cell proliferation and IL-8 secretion from HCT8 cells were investigated. Furthermore, the association between MAPK pathway activation and the inhibitory effects of SN38 on cell proliferation and IL-8 upregulation in HCT8 cells was also investigated, in an aim to elucidate the mechanisms of action of SN38 in colon cancer.

\section{Materials and methods}

Materials and chemicals. SN38 (purity, 99.4\%) was purchased from Selleck Chemicals (Houston, TX, USA). Evodiamine (Evo; purity, 98\%), Jatrorrhizine (Jat; purity, 98\%) and 5-fluorouracil (5-Fu; purity, 98\%) were obtained from Melonepharma (Dalian, China). Trypsin and fetal bovine 
serum (FBS) were provided by Gibco (Grand Island, NY, USA). The cell counting kit-8 (CCK-8) was provided by Dojindo Laboratories (Kumamoto, Japan). The IL-8 enzyme-linked immunosorbent assay (ELISA) kit was obtained from eBioscience (San Diego, CA, USA). Anti-p-p38 MAPK (Cat. no. 9211), anti-p-extracellular signal-regulated kinase (ERK)1/2 (Cat. no. 9101), anti-p-c-Jun N-terminal kinase (JNK; Cat.no.9251), anti-p-Januskinase 2(Jak2; Cat. no.3771), anti-Jak2 (Cat. no. 3773), anti-p-signal transducer and activator of transcription 3 (STAT3; Cat. no. 9131), anti-STAT3 (Cat. no. 9132), anti-p38 MAPK (Cat. no. 9212), anti-ERK1/2 (Cat. no. 9102), anti-JNK (Cat. no. 9252) and anti- $\beta$-actin (Cat. no. 3700) antibodies were supplied by Cell Signaling Technology (Danvers, MA, USA). The ECL prime kit was purchased from GE Healthcare (Little Chalfont, UK). SB202190, SP600125, and PD98059 were purchased from Selleck Chemicals. Anisomycin was obtained from Calbiochem (La Jolla, CA, USA).

Cell culture. The HCT8 cells obtained from the Cell Bank of Type Culture Collection of Chinese Academy of Sciences and cultured in RMPI-1640 medium (Thermo Fisher Scientific,Inc., Waltham, MA, USA), supplemented with 10\% FBS. The cells were cultured at $37^{\circ} \mathrm{C}$ in a humidified incubator with $5 \% \mathrm{CO}_{2}$.

Cell proliferation assay. The cells were seeded in $100 \mu \mathrm{l}$ of medium at $1.0 \times 10^{5}$ cells $/ \mathrm{ml}$ in 96-well culture plates and grown overnight. The cells were treated with SN38 $(0.4-2,000 \mathrm{ng} / \mathrm{ml})$, Jat (1-400 $\mu \mathrm{M}), 5-\mathrm{Fu}(30 \mu \mathrm{M})$, Evo $(0.1-100 \mu \mathrm{M})$, or a combination of Jat $(50$ and $100 \mu \mathrm{M})$ and SN38 (40 and $80 \mathrm{ng} / \mathrm{ml})$ for 24 or $48 \mathrm{~h}$. The medium was then removed and replaced with an equal volume of fresh medium with an additional $10 \mu \mathrm{l}$ of CCK-8 solution followed by incubation at $37^{\circ} \mathrm{C}$ for a further $30 \mathrm{~min}$. The absorbance of the dissolved solutions was detected at $450 \mathrm{~nm}$ using a Thermo Scientific Varioskan Flash microplate reader (Thermo Fisher Scientific, Inc.). The cell viability rate was calculated as follows: (absorbance of drug-treated sample/absorbance of control sample) x100.

To identify the involvement of MAPKs in the modulation of cell proliferation, following pre-treatment with or without inhibitors of p38 MAPK (SB202190, $25 \mu \mathrm{M}$ ), ERK1/2 (PD98059, $20 \mu \mathrm{M}$ ) and JNK (SP600125, $20 \mu \mathrm{M}$ ), and the activator of MAPKs (anisomycin, $2.5 \mathrm{ng} / \mathrm{ml}$ ) for $1 \mathrm{~h}$, the cells were treated with or without SN38 (40 ng/ml) for 24 or $48 \mathrm{~h}$. Cell viability was then measured by CCK- 8 assay.

ELISA. For in vitro experiments, the HCT8 cells were seeded in 96-well culture plates and cultured overnight. Following treatment with or without various concentrations of SN38, Evo, 5-Fu and Jat for $48 \mathrm{~h}$, the culture medium was collected and subjected to IL-8 assay using a respective kit. To identify the involvement of MAPKs in the modulation of IL-8 expression, following pre-treatment with SB202190 $(25 \mu \mathrm{M})$, SP600125 $(20 \mu \mathrm{M})$, PD98059 $(20 \mu \mathrm{M})$ and anisomycin $(2.5 \mathrm{ng} / \mathrm{ml})$ for $1 \mathrm{~h}$, the cells were treated with or without SN38 $(40 \mathrm{ng} / \mathrm{ml})$ for 24 or $48 \mathrm{~h}$. The culture medium was then collected for IL-8 assay.

Western blot analysis. The cells were collected and lysed with cell lysis buffer and sonicated 3 times, each for $15 \mathrm{sec}$. The lysate was centrifuged at $14,000 \mathrm{x} \mathrm{g}$ for $15 \mathrm{~min}$ at $4^{\circ} \mathrm{C}$, and the supernatant was collected. Protein samples were separated by sodium dodecyl sulfate-polyacrylamide gel electrophoresis (SDS-PAGE) and transferred onto PVDF membranes by the wet transfer approach. The PVDF membranes were then blocked with 5\% non-fat milk solution and incubated with various primary antibodies overnight at $4^{\circ} \mathrm{C}$. After being washed with 1X PBST, the PVDF membranes were incubated with the respective secondary antibodies. The protein bands were visualized using the ECL-prime kit.

Statistical analysis. Each value represents the mean \pm SE. Differences among groups were analyzed by one-way ANOVA with Dunnett's Test using PrismDemo 5 software. A value of $\mathrm{P}<0.05$ was considered to indicate a statistically significant difference.

\section{Results}

Use of different chemical compounds results in differential effects on IL-8 secretion from HCT8 cells. In our experiments (Fig. 1), following treatment with Jat, SN38, 5-Fu and Evo for 24 or $48 \mathrm{~h}$, SN38 induced a significant increase in IL-8 secretion from HCT8 cells $(\mathrm{P}<0.001$ vs. control). However, 48-h treatment with Evo $(0.1 \mu \mathrm{M})$ suppressed IL-8 secretion $(\mathrm{P}<0.001$ vs. control). In addition, although treatment with Jat $(50$ and $100 \mu \mathrm{M})$ alone did not have a significant effect on IL-8 secretion, the combination of Jat and SN38 significantly increased IL-8 secretion ( $\mathrm{P}<0.001$ vs. control), compared to treatment with SN38 (40 and $80 \mathrm{ng} / \mathrm{ml}$ ) alone. Treatment with 5-Fu also increased IL-8 secretion, but without any significant difference.

In order to confirm the effects of Jat, SN38 and Evo on IL-8 secretion from HCT8 cells, we further investigated the effects of Jat, SN38 and Evo at various concentrations on IL-8 secretion from and the viability of HCT8 cells. As shown in Fig. 2, SN38 dose-dependently inhibited cell viability $(\mathrm{P}<0.001$ vs. control), but significantly increased IL-8 secretion in a dose-dependent manner $(\mathrm{P}<0.001$ vs. control). Jat had no obvious effect on cell viability, but enhanced IL-8 secretion from HCT8 cells $(\mathrm{P}<0.01$ and $\mathrm{P}<0.001$ vs. control). In addition, Evo significantly inhibited the viability of HCT8 cells; however, it only inhibited IL-8 secretion at a very low concentration $(0.1 \mu \mathrm{M})$ ( $\mathrm{P}<0.01$ vs. control).

SN38 activates the MAPK pathways in HCT8 cells. To determine the effects of SN38 on MAPK pathways, including the p38 MAPK, ERK1/2 and JNK pathways in HCT8 cells, western blot analysis was conducted. Treatment with SN38 at $40 \mathrm{ng} / \mathrm{ml}$ for $24 \mathrm{~h}$ increased the phosphorylation of p38 MAPK, ERK1/2 and JNK, then SN38 at $200 \mathrm{ng} / \mathrm{ml}$ increased the phosphorylation of p38 MAPK and ERK1/2, but not that of JNK (Fig. 3).

SN38 inhibits the activation of the Jak2 pathway in HCT8 cells. In our experiments, we also determined the effects of SN38 on the Jak2-STAT3 pathway. As shown in Fig. 4, to our surprise, the results revealed that treatment with SN38 at $80 \mathrm{ng} / \mathrm{ml}$ significantly decreased the phosphorylation of Jak2, whereas it had no significant effect on the phosphorylation of STAT3.

Upregulation of IL-8 secretion induced by SN38 does not affect its inhibitory effect on the viability of HCT8 cells. In 

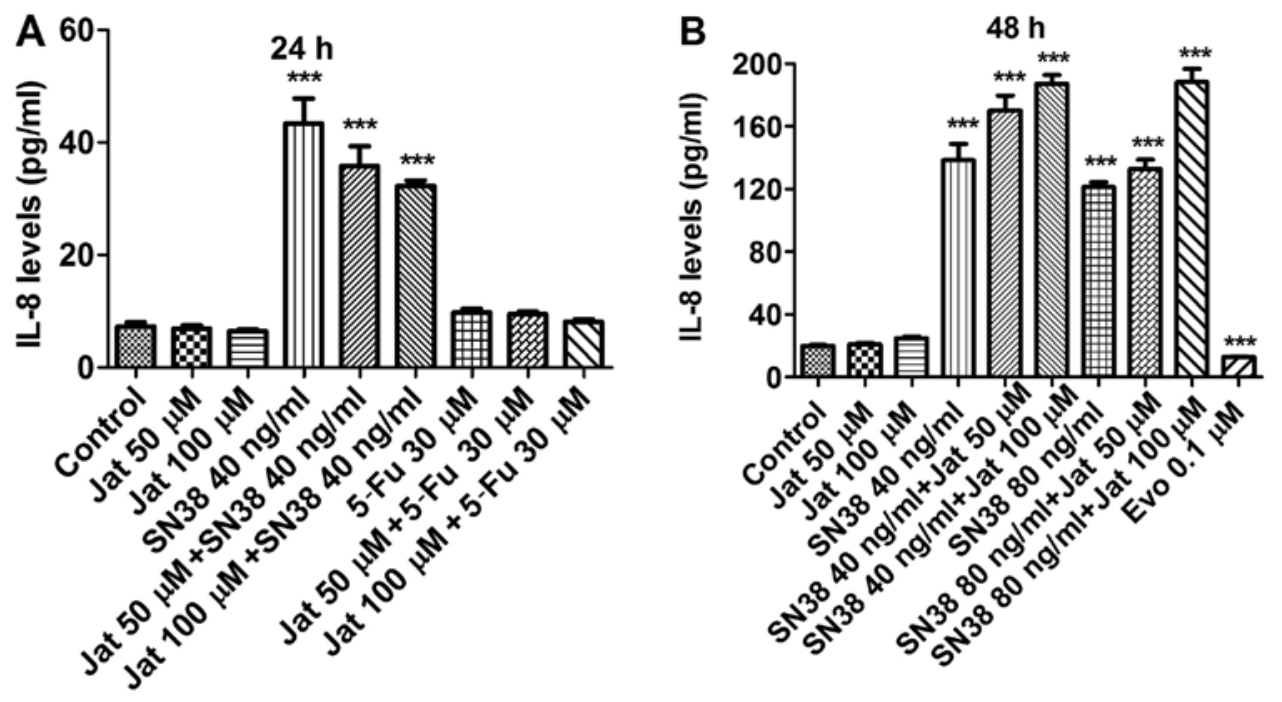

Figure 1. Effect of jatrorrhizine (Jat), evodiamine (Evo), 5-fluorouracil (5-Fu) and SN38 on interleukin-8 (IL-8) secretion of HCT8 cells. (A) Treatment wiht SN38 for $24 \mathrm{~h}$ increased IL- 8 secretion, which was measured by ELISA; (B) SN38 and the combination of SN38 with Jat for $48 \mathrm{~h}$ increased IL-8 secretion, which was measured by ELISA. Data represent the means \pm SEM, ${ }^{* * *} \mathrm{P}<0.001$ vs. control.
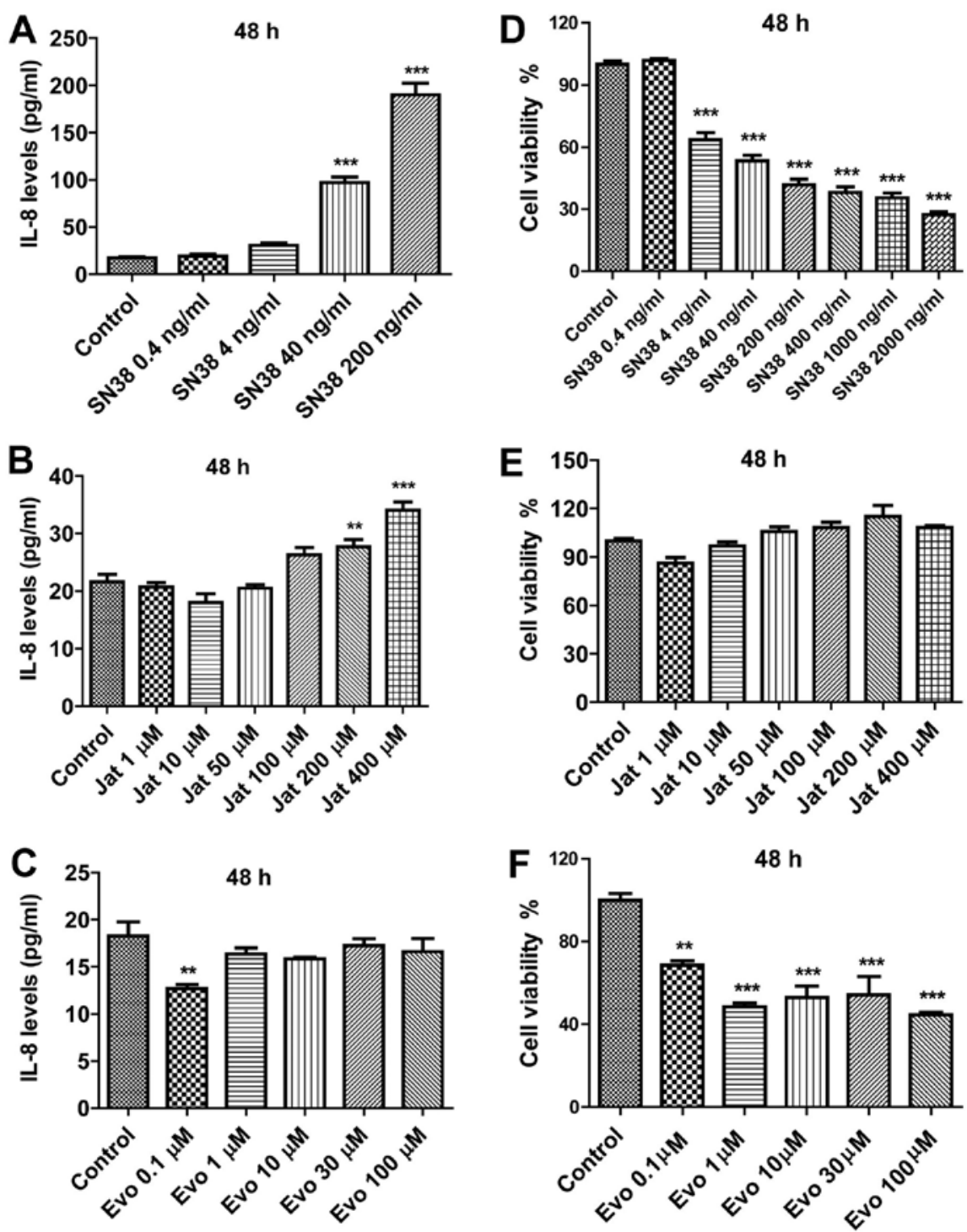

Figure 2. Effect of jatrorrhizine (Jat), evodiamine (Evo) and SN38 on interleukin-8 (IL-8) secretion and the viability of HCT8 cells. (A-C) Effect of SN38, Jat and Evo on IL-8 secretion from HCT8 cells, which was detected by ELISA. (D-F) Effect of SN38, Jat and Evo on the viability of HCT8 cells, which was measured by CCK-8 assay. Data represent the means $\pm \mathrm{SEM},{ }^{* *} \mathrm{P}<0.01$ and ${ }^{* * * *} \mathrm{P}<0.001$ vs. control. 


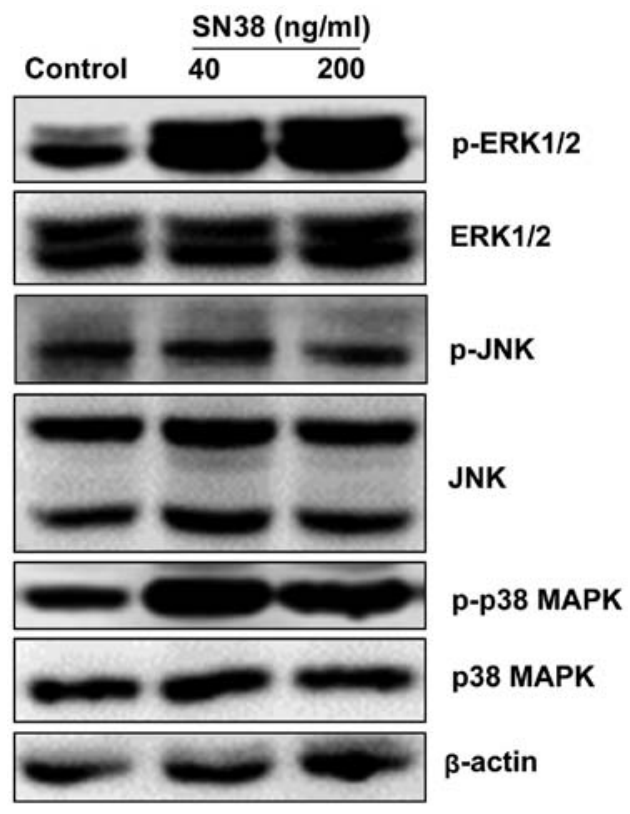

Figure 3. Effect of SN38 on the phosphorylation of p38 MAPK, ERK1/2 and JNK in HCT8 cells. Treatment with SN38 for $24 \mathrm{~h}$ inhibited the intracellular phosphorylation of p38 MAPK, ERK1/2 and JNK, which was detected by western blot analysis.

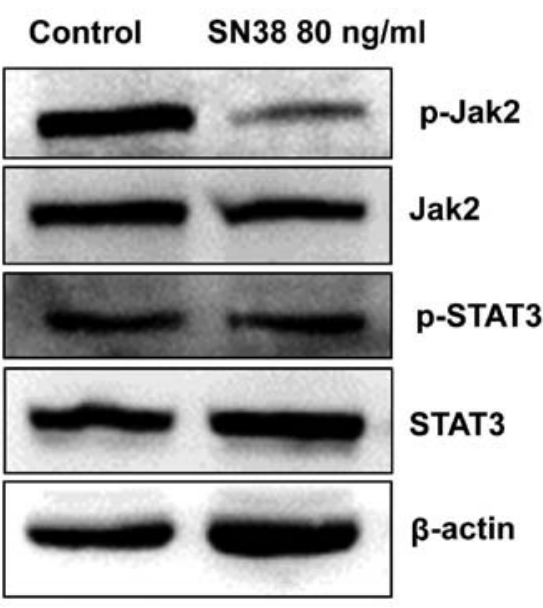

Figure 4. Effect of SN38 on the phosphorylation of Jak2 and STAT3 in HCT8 cells. Treatment with SN38 for 24 h inhibited the intracellular phosphorylation of Jak2, which was detected by western blot analysis.

order to further clarify whether the MAPK pathways are involved in cell proliferation, and whether SN38 inhibits the proliferation of colon cancer cells through the activation of MAPKs, the inhibitors of p38 MAPK (SB202190), JNK (SP600125) and ERK1/2 (PD98059) were used in CCK-8 assay. As shown in Fig. 5, in the HCT8 cells, treatment with these inhibitors enhanced the inhibitory effects of SN38 on cell proliferation $(\mathrm{P}<0.001$ vs. control). To our surprise, not only anisomycin, the activator of p38 MAPK and JNK, but also the inhibitors of MAPKs, all significantly decreased the viability of the HCT8 cells $(\mathrm{P}<0.01$ and $\mathrm{P}<0.001$ vs. control) (Fig. 5A and $\mathrm{B})$.

SN38 increases IL-8 secretion by enhancing the phosphorylation of MAPKs in HCT8 cells. In a previous study, SN38 was found to increase IL-8 expression in a dose- and time- dependent manner in AGS cells (16). However, whether the promoting effect of SN38 on IL-8 expression is cell-type dependent remains unknown. In this study, as shown in Figs. 1, 2 and 5, SN38 was also found to increase the secretion of IL- 8 from HCT8 cells $(\mathrm{P}<0.001$ vs. control). Further experiments revealed that anisomycin significantly increased the secretion of IL-8, but reduced the viability of HCT8 cells $(\mathrm{P}<0.001$ vs. control); however, treatment with anisomycin in combination with SN38 completely enhanced IL-8 secretion ( $\mathrm{P}<0.01$ vs. anisomycin) (Fig. 5). However, treatment with SB202190, SP600125 or PD98059 significantly decreased IL-8 secretion from HCT8 cells $(\mathrm{P}<0.001$ vs. control), and also decreased IL-8 secretion induced by SN38 (Fig. 5C).

\section{Discussion}

Previous studies have demonstrated that IL-8 is closely associated tumorigenesis and the metastasis of colon cancer, as well as that of gastric cancer and breast cancer (8-10). Over the past years, many chemotherapeutic drugs have been proven to induce IL-8 overexpression in a number of cancer cells in vitro and in vivo (13-17), indicating a potential risk of promoting cancer metastasis during the treatment period or post-treatment period in clinic practice. However, the effects and molecular mechanisms of action of SN38 on IL-8 expression have not yet been fully clarified, particularly as regards colon cancer. In this study, we found that SN38 significantly enhanced IL-8 secretion from HCT 8 cells by promoting the activation of the MAPK pathways.

It is well known that many chemotherapeutic agents can induce the upregulation of IL-8 in tumor cells, which is closely associated with resistance to chemotherapy and cancer metastasis $(7,9,15,16,20,21)$. The overexpression of IL-8 in cancer cells has a wide significance to the tumor microenvironment through CXCR1 and CXCR2 receptors on tumor cells, neutrophils/tumor-associated macrophages and endothelial cells, and even promotes angiogenesis and metastasis $(3,6,7,17)$. By contrast, the depletion of IL- 8 induces cell cycle arrest and increases the efficacy of chemotherapeutic agents in breast cancer cells (22). In our experiments, it was very interesting that the induction of IL-8 expression by some compounds may be cell type-dependent and/or tissue type dependent. We observed the effects of 4 compounds, namely Jat, Evo, 5-Fu and SN38, on IL-8 expression in HCT8 cells, and the results indicated that only SN38 significantly enhanced IL-8 secretion from HCT8 cells. Evo, which has been found to significantly induce IL-8 expression in AGS cells (9), even decreased IL-8 secretion from HCT8 cells. 5-Fu, which has also been proven to increase IL-8 expression in breast cancer cells (14), had no significant effect on IL-8 secretion from HCT8 cells. Berberine, a major alkaloid molecule isolated from the Chinese herbal plant, Coptis chinensis Franch. Huanglian (C. chinensis), has been shown to inhibit the proliferation, as well as the expression of IL-8 in AGS cells and MDA-MB-231 cells $(9,10,23)$. However, in our study, jatrorrhizine, another active compound isolated from $C$. chinensis, can increase IL-8 secretion at high doses (200 and $400 \mu \mathrm{M}$ ) (Fig. 2B). Although previous studies found that the upregulation of IL- 8 was associated with the proliferation of colon cancer cells $(11,24)$, we found that the proliferation of of HCT8 cells was independent of IL-8. SN38 

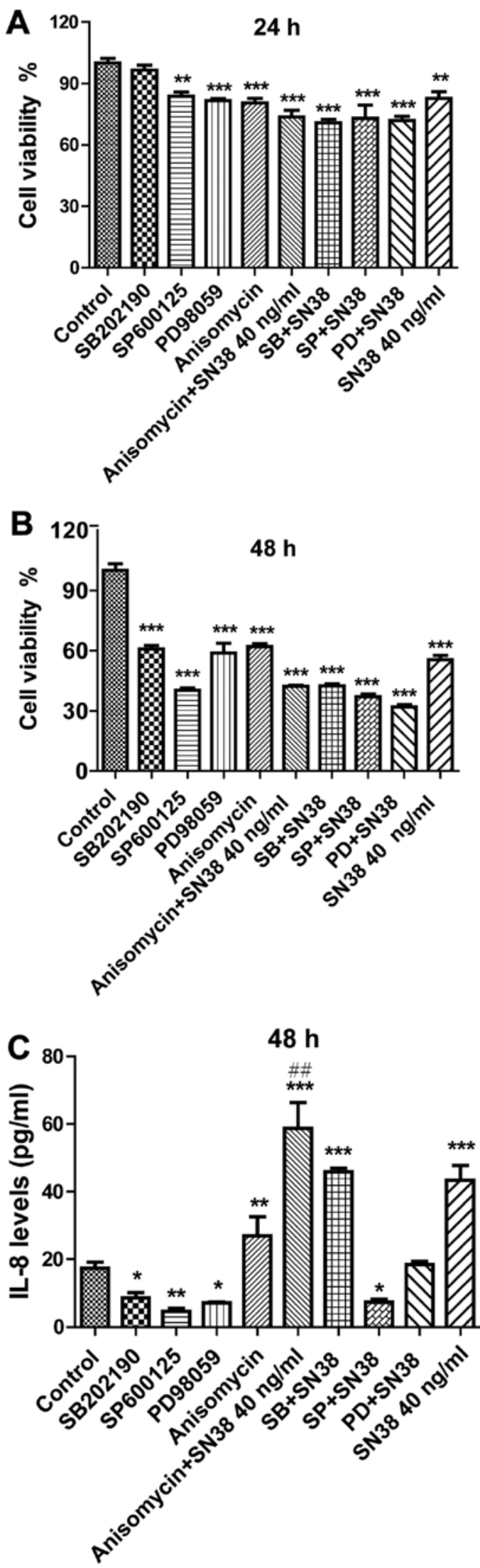

Figure 5. MAPKs are involved in the inhibitory effects of SN38 on (A and B) the proliferation of HCT 8 cells for 24 and $48 \mathrm{~h}$ and $(\mathrm{C})$ the upregulation of interleukin-8 (IL-8) secretion. Cell viability was detected by CCK-8 assay and the IL- 8 content was measured by ELISA. SB202190 (SB): the inhibitor of p38 MAPK, $25 \mu \mathrm{M}$; SP600125 (SP): the inhibitor of JNK, $20 \mu \mathrm{M}$; PD98059 (PD): the inhibitor of ERK1/2, $20 \mu \mathrm{M}$; anisomycin: the activator of p38 MAPK and JNK, $0.05 \mu \mathrm{g} / \mathrm{ml}$. Data represent the means $\pm \mathrm{SEM},{ }^{*} \mathrm{P}<0.05$, ${ }^{* * *} \mathrm{P}<0.01$ and ${ }^{* * * *} \mathrm{P}<0.001$ vs. control; ${ }^{\# \#} \mathrm{P}<0.01$ vs. anisomycin. significantly increased IL- 8 secretion, but inhibited the proliferation of HCT8 cells in a dose-dependent manner.

SN38 is the metabolic active compound of CPT-11, which is a first-line chemotherapeutic drug used in the treatment of patients with colon cancer. In a previous study, SN38 was shown to increase IL-8 expression in the AGS gastric cancer cell line (16). Although the induction of IL- 8 by compounds was cell-type dependent, SN38 increased IL-8 expression both in AGS cells (16) and, as shown in this study, in HCT8 cells in a dose-dependent manner.

Further experiments demonstrated that SN38 markedly enhanced the phosphorylation of ERK1/2,JNK and p38 MAPK in HCT8 cells. However, to our surprise, SN38 significantly decreased the phosphorylation of Jak2, whereas SN38 had no significant effect on the phosphorylation of STAT3. In order to further clarify the association between MAPK pathways and IL-8 secretion and cell proliferation, the inhibitors and activator of MAPK pathway were used. MAPKs have been disclosed to be actively involved in the modulation of IL-8 production $(9,10,13,17,25-28)$. In agreement with these studies, our results indicated that the activation of the ERK1/2, JNK and p38 MAPK pathways was closely associated with the constitutive IL-8 secretion from HCT8 cells. On the one hand, inhibitors of p38 MAPK, JNK and ERK significantly decreased IL-8 expression from HCT8 cells. On the other hand, when the MAPK agonist, anisomycin, was applied, IL-8 secretion was enhanced.

SN38, similar to the MAPK activator, enhanced the activation of ERK1/2, JNK and p38 MAPK and increased IL-8 secretion, which was counteracted by the inhibitors of MAPKs. To our surprise, not only enhancing, but also decreasing the activation of MAPKs both decreased cell viability since anisomycin and SB202190, SP600125 and PD98059 all inhibited the proliferation of HCT8 cells. However, similar to our findings, curcumin has been reported to induce apoptosis through the activation of ERK1/2 in AGS cells (29), while apoptosis has been shown to be enhanced by capsaicin by the inhibition of MAPKs in AGS cells (30). These results reflect the complex functions of MAPKs in cancer cells, indicating the importance of MAPK pathways in the proliferation of colon cancer cells. Although the activation of Jak2 has been proven to be involved in IL-8 expression in AGS cells (9), in this study, SN38 significantly inhibited the phosphorylation of Jak2, thus indicating that the Jak2 signaling pathway was not involved in the IL-8 secretion induced by SN38 in HCT8 cells.

In conclusion, SN38 inhibited the growth of HCT8 cells in an IL-8 independent manner, and significantly increased IL-8 expression by promoting the activation of p38 MAPK, ERK1/2 and JNK pathways, but that of Jak2 independently. All of our findings suggested the potential combination of SN38 with other drugs with inhibitory effects on IL- 8 expression in the therapy of colon cancer in clinical practice to increase the safety and efficacy of SN38. Whether Evo could counteract the effect of SN38 on IL-8 secretion warrents further investigation in future studies.

\section{Acknowledgements}

This study was supported by the Youth Foudation of Zhongshan Hospital Fudan University (grant no. 2014ZSQN39). 


\section{References}

1. Kuai WX, Wang Q, Yang XZ, Zhao Y, Yu R and Tang XJ: Interleukin-8 associates with adhesion, migration, invasion and chemosensitivity of human gastric cancer cells. World $\mathbf{J}$ Gastroenterol 18: 979-985, 2012.

2. Bünger S, Haug U, Kelly FM, Klempt-Giessing K, Cartwright A, Posorski N, Dibbelt L, Fitzgerald SP, Bruch HP, Roblick UJ, et al BMBF-Consortium ‘Colorectal Cancer Screening Chip': Toward standardized high-throughput serum diagnostics: Multiplexprotein array identifies IL-8 and VEGF as serum markers for colon cancer. J Biomol Screen 16: 1018-1026, 2011.

3. Kitadai Y, Takahashi Y, Haruma K, Naka K, Sumii K, Yokozaki H, Yasui W, Mukaida N, Ohmoto Y, Kajiyama G, et al: Transfection of interleukin- 8 increases angiogenesis and tumorigenesis of human gastric carcinoma cells in nude mice. $\mathrm{Br}$ J Cancer 81: 647-653, 1999.

4. Kido S, Kitadai Y, Hattori N, Haruma K, Kido T, Ohta M, Tanaka S, Yoshihara M, Sumii K, Ohmoto Y and Chayama K Interleukin 8 and vascular endothelial growth factor - prognostic factors in human gastric carcinomas? Eur J Cancer 37: 14821487, 2001.

5. Xie K: Interleukin-8 and human cancer biology. Cytokine Growth Factor Rev 12: 375-391, 2001

6. Matsuo Y, Ochi N, Sawai H, Yasuda A, Takahashi H, Funahashi H, Takeyama H, Tong Z and Guha S: CXCL8/IL-8 and CXCL12/SDF-1alpha co-operatively promote invasiveness and angiogenesis in pancreatic cancer. Int J Cancer 124: 853-861, 2009.

7. Ju D, Sun D, Xiu L, Meng X, Zhang C and Wei P: Interleukin-8 is associated with adhesion, migration and invasion in human gastric cancer SCG-7901 cells. Med Oncol 29: 91-99, 2012.

8. Asfaha S, Dubeykovskiy AN, Tomita H, Yang X, Stokes S, Shibata W, Friedman RA, Ariyama H, Dubeykovskaya ZA, Muthupalani S, et al: Mice that express human interleukin-8 have increased mobilization of immature myeloid cells, which exacerbates inflammation and accelerates colon carcinogenesis. Gastroenterology 144: 155-166, 2013.

9. Shi HL, Wu XJ, Liu Y and Xie JQ: Berberine counteracts enhanced IL-8 expression of AGS cells induced by evodiamine. Life Sci 93: 830-839, 2013.

10. Li X, Zhao SJ, Shi HL, Qiu SP, Xie JQ, Wu H, Zhang BB, Wang ZT, Yuan JY and Wu XJ: Berberine hydrochloride IL-8 dependently inhibits invasion and IL-8-independently promotes cell apoptosis in MDA-MB-231 cells. Oncol Rep 32: 2777-2788, 2014.

11. Cacev T, Radosević S, Krizanac S and Kapitanović S: Influence of interleukin-8 and interleukin-10 on sporadic colon cancer development and progression. Carcinogenesis 29: 1572-1580, 2008.

12. Lee YS, Choi I, Ning Y, Kim NY, Khatchadourian V, Yang D, Chung HK, Choi D, LaBonte MJ, Ladner RD, et al: Interleukin-8 and its receptor CXCR2 in the tumour microenvironment promote colon cancer growth, progression and metastasis. Br J Cancer 106: 1833-1841, 2012.

13. Collins TS, Lee LF and Ting JP: Paclitaxel upregulates interleukin-8 synthesis in human lung carcinoma through an NF-kappaB- and AP-1-dependent mechanism. Cancer Immunol Immunother 49: 78-84, 2000.

14. De Larco JE, Wuertz BR, Manivel JC and Furcht LT: Progression and enhancement of metastatic potential after exposure of tumor cells to chemotherapeutic agents. Cancer Res 61: 2857-2861, 2001.
15. Lev DC, Onn A, Melinkova VO, Miller C, Stone V, Ruiz M, McGary EC, Ananthaswamy HN, Price JE and Bar-Eli M: Exposure of melanoma cells to dacarbazine results in enhanced tumor growth and metastasis in vivo. J Clin Oncol 22: 2092-2100, 2004.

16. Kishida O, Miyazaki Y, Murayama Y, Ogasa M, Miyazaki T, Yamamoto T, Watabe K, Tsutsui S, Kiyohara T, Shimomura I, et al: Gefitinib (Iressa, ZD1839) inhibits SN38-triggered EGF signals and IL-8 production in gastric cancer cells. Cancer Chemother Pharmacol 55: 584-594, 2005.

17. Waugh DJ and Wilson C: The interleukin-8 pathway in cancer. Clin Cancer Res 14: 6735-6741, 2008.

18. Bleiberg H: CPT-11 in gastrointestinal cancer. Eur J Cancer 35: 371-379, 1999.

19. Ajani JA, Baker J, Pisters PW, Ho L, Mansfield PF, Feig BW and Charnsangavej C: CPT-11 plus cisplatin in patients with advanced, untreated gastric or gastroesophageal junction carcinoma: Results of a phase II study. Cancer 94: 641-646, 2002.

20. Britschgi A, Andraos R, Brinkhaus H, Klebba I, Romanet V, Müller U, Murakami M, Radimerski T and Bentires-Alj M: JAK2/STAT5 inhibition circumvents resistance to PI3K/mTOR blockade: A rationale for cotargeting these pathways in metastatic breast cancer. Cancer Cell 22: 796-811, 2012.

21. Zhang W, Feng M, Zheng G, Chen Y, Wang X, Pen B, Yin J, Yu Y and He Z: Chemoresistance to 5-fluorouracil induces epithelialmesenchymal transition via upregulation of Snail in MCF7 human breast cancer cells. Biochem Biophys Res Commun 417: 679-685, 2012.

22. Shao N, Chen LH, Ye RY, Lin Y and Wang SM: The depletion of interleukin-8 causes cell cycle arrest and increases the efficacy of docetaxel in breast cancer cells. Biochem Biophys Res Commun 431: 535-541, 2013.

23. Shi HL, Xie JQ and Wu DZ: Effect of berberine on cell proliferation and IL-8 expression in AGS cells. Pharmacol Clin Chin Mater Med Chin 28: 45-48, 2012.

24. Itoh Y, Joh T, Tanida S, Sasaki M, Kataoka H, Itoh K, Oshima T, Ogasawara N, Togawa S, Wada T, et al: IL-8 promotes cell proliferation and migration through metalloproteinase-cleavage proHB-EGF in human colon carcinoma cells. Cytokine 29: 275-282, 2005 .

25. Chelouche-Lev D, Miller CP, Tellez C, Ruiz M, Bar-Eli M and Price JE: Different signalling pathways regulate VEGF and IL-8 expression in breast cancer: Implications for therapy. Eur J Cancer 40: 2509-2518, 2004.

26. Maxwell PJ, Gallagher R, Seaton A, Wilson C, Scullin P, Pettigrew J, Stratford IJ, Williams KJ, Johnston PG and Waugh DJ: HIF-1 and NF-kappaB-mediated upregulation of CXCR1 and CXCR2 expression promotes cell survival in hypoxic prostate cancer cells. Oncogene 26: 7333-7345, 2007.

27. Bezzerri V, Borgatti M, Finotti A, Tamanini A, Gambari R and Cabrini G: Mapping the transcriptional machinery of the IL-8 gene in human bronchial epithelial cells. J Immunol 187: 6069-6081, 2011

28. Ning Y and Lenz HJ: Targeting IL-8 in colorectal cancer. Expert Opin Ther Targets 16: 491-497, 2012.

29. Cao AL, Tang QF, Zhou WC, Qiu YY, Hu SJ and Yin PH: Ras-ERK signaling pathway is involved in curcumin-induced cell cycle arrest and apoptosis in human gastric carcinoma AGS cells. J Asian Nat Prod Res 17: 56-63, 2015.

30. Park SY, Kim JY, Lee SM, Jun CH, Cho SB, Park CH, Joo YE, Kim HS, Choi SK and Rew JS: Capsaicin induces apoptosis and modulates MAPK signaling in human gastric cancer cells. Mol Med Rep 9: 499-502, 2014. 